\section{Italian Chronic Cerebrospinal Venous Insufficiency National Epidemiological Observatory methodology and preliminary data}

Sandro Mandolesi, ${ }^{1}$ Aldo d'Alessandro, ${ }^{2}$ Marco Matteo Ciccone, ${ }^{3}$ Annapaola Zito, ${ }^{3}$ Tarcisio Niglio, ${ }^{4}$ Ettore Manconi, ${ }^{5}$

Dimitri Mandolesi, ${ }^{6}$

Alessandro d'Alessandro, ${ }^{7}$ Aldo Bruno, ${ }^{8}$

Francesco Fedele ${ }^{1}$

'Department of Cardiovascular and

Respiratory Sciences, La Sapienza

University, Roma; ' ${ }^{2}$ Department of

Neuroscience, Imaging and Clinical

Sciences, G. d'Annunzio University, Chieti-Pescara; ${ }^{3}$ Cardiovascular Diseases Section, Department of Emergency and Organ Transplantation, University of Bari; ${ }^{4}$ Higher Health Institute, Roma; ${ }^{5}$ Department of Cardiovascular and Neurological Sciences, University of Cagliari; ${ }^{6}$ Occupational Medicine, La Sapienza University, Roma; ${ }^{7}$ Faculty of Medicine, University of Foggia; ${ }^{8}$ Department of Vascular Surgery, Clinica Gepos, Telese Terme (BN), Italy

\section{Abstract}

The aim of our work is to describe the Memnet program's use and potential and to show the data of Italian Chronic Cerebrospinal Venous Insufficiency (CCSVI)-National Epidemiological Observatory (NEO) activity in the first three years (http:/www.osservatorioccsvi.org). From 2011 to 2014, all echo-colorDoppler (ECD) assessments were stored by Mem-net program into CCSVI-NEO web site (http://www.mem-net.it). Mem-net is a tool for multicenter data collection based on the International Society for Neurovascular Disease consensus and position statement, where we can insert patients (pts) history, neurological visits, ECD assessments, different examinations, therapies and surgical procedures. The website provides an epidemiological and statistical program for data analysis in real time. At present, 7 medical centers, affiliated to CCSVI-NE0, input their symptomatic and asymptomatic subjects with CCSVI. Data were storage using the Mem-net program.

We analyzed data of only four centers on seven (Rome, Bari, Cagliari and Benevento). Total pts number with multiple sclerosis (MS) was 1109 , mean age $46.0 \pm 13.4$ [male 422 (38.05\%); female 687 (61.95\%)]. CCSVI posi- tive pts were 937 (84.49\%), CCSVI negative pts were 172 (15.51\%). The CCSVI type 1 subjects were 530 (56.56\%), CCSVI type 2 subjects were 20 (2.13\%), CCSVI type 3 subjects were 387 (41.30\%). We found 800 (85.38\%) pts with criterion $1 ; 725(77.37 \%)$ with criterion $2 ; 519$ (55.39\%) with criterion $3 ; 483$ (51.55\%) with criterion $4 ; 88(9.39 \%)$ with criterion 5 . The venous hemodynamic insufficiency severity score mean score was 3.8 ; the CCSVI mean score was 2.8; the MEM mean score was 34.7 ; the expanded disability status scale mean score was 4.5 ; the disease mean duration was $12.5 \pm 5.7$ years. MS clinical types were divided as follows: relapsing-remitting pts were 449 (47.92\%), Secondary progressive pts were 144 (15.37\%), primary progressive pts were 72 (7.68\%). The CCSVI-NEO database and Memnet software may be useful medical and researching tools for recording, storing, analyzing and studying ECD and vascular data. Preliminary data of NEO show an elevated prevalence of CCSVI in MS.

\section{Introduction}

Chronic cerebrospinal venous insufficiency (CCSVI) is characterized by multiple stenosis/obstructions affecting the principal extracranial outflow pathways of the cerebrospinal venous system, the internal jugular veins (IJVs), the vertebral veins (VVs) and the azygos vein, distributed in four main hemodynamic patterns. ${ }^{1-3}$ Furthermore, CCSVI determines significant changes in cerebral venous hemodynamic, with a very high incidence of reflux in both intra- and extra-cranial venous segments as well as loss of the postural regulation of cerebral venous outflow. ${ }^{4-6}$ Zamboni suggested five echo-color-Doppler (ECD) venous criteria for this syndrome. ${ }^{1-3}$ criterion 1 : constantly reflux or bidirectional present in an outflow pathway in supine and up-right; criterion 2: reflux propagated upward to the deep cerebral veins, including internal cerebral vein, basal vein, Galen vein, criterion 3: evidence of IJVs anatomical stenosis or presence of anomalies and block, reflux constantly or bidirectional in the other, criterion 4: flow no detectable in the IJVs and VVs in clino and upright or in one position and presence of anomalies and reflux constantly or bidirectional in the other, criterion 5: negative difference in cross sectional area of the IJV (CSA) assessed in supine and standing posture $\left(0^{\circ}\right.$ and $\left.90^{\circ}\right)$ in the IJV ( CSA). The presence of two of them is enough to diagnose CCSVI. ${ }^{1-2}$ These criteria for ECD diagnosis of CCSVI proposed by Zamboni in 2009 was approved in 2011 by a consensus of experts of seven international scientific societies of vascular area. ${ }^{3}$

The aim of our work is to describe the Mem-
Correspondence: Sandro Mandolesi, via Montebello 17, 00185 Roma, Italy.

Tel: +39.335 .6512303 - Fax: +39.06 .4873984 .

E-mail: s.mandolesi@email.it

Key words: chronic cerebrospinal venous insufficiency (CCSVI), CCSVI National Epidemiological Observatory, Mem-net, multiple sclerosis.

Conflict of interest: all authors declare to have no financial associations that might pose a conflict of interest.

Received for publication: 16 September 2014. Revision received: 3 February 2015.

Accepted for publication: 5 February 2015.

This work is licensed under a Creative Commons Attribution 3.0 License (by-nc 3.0).

(C) Copyright S. Mandolesi et al., 2014

Licensee PAGEPress, Italy

Veins and Lymphatics 2014; 3:4707

doi:10.4081/vl.2014.4707

net program's use and potential and to show the data of Italian CCSVI-National Epidemiological Observatory (NEO) activity in the first three years. The Italian CCSVI-NEO has all database stored at http://www.osservatorioccsvi.org.

\section{Materials and Methods}

The Department of Cardiovascular, Respiratory and Morphologic Sciences of Umberto I Polyclinic of Rome, La Sapienza University, developed in 2010 the NE0 and in 2011 it made the digitalized platform CCSVIdatabase: the Mem-net program. Mem-net is a tool for multicenter data collection based on the International Society for Neurovascular Disease (ISNVD) consensus and position statement. $^{3}$ This software is accessible at http://www.mem-net.it. In this software we can insert patient history, neurological visit, ECD assessment of brain veins, magnetic resonance imaging, venography examination, interventional procedure, physical therapy and surgical procedures. The website provides an epidemiological and statistical program for data analysis in real time.

At present, seven medical centers, affiliated to CCSVI-NEO, input their symptomatic and asymptomatic CCSVI subjects' data. The ECD assessments of MS symptomatic and asymptomatic patients were stored on years from 2011 to 2014 using Mem-net program. In this study we analyzed data of only four centers on seven (Rome, Bari, Cagliari and Benevento). All the sonographers of these centers received a 
course to use the Mem-net program. We studied 1109 patients suffering from multiple sclerosis, which was diagnosed according to the revised McDonald's criteria. ${ }^{7,8}$ The study did not include patients having undergone an acute relapse and/or steroid treatment within the 30 days before the enrollment, suffering from preexisting clinical conditions related to brain pathology, systemic vasculitis, vascular malformations of the brain (i.e. arterio-venous and cavernous malformations, venous hemangioma, telangiectasias) and congenital malformations leading to hydrocephalus (i.e. ArnoldChiari syndrome, aqueduct atresia or stenosis and Dandy-Walker disease). A full clinical evaluation of each patient was performed by one expert physician in order to assess age, gender, onset of disease symptoms, clinical variants of multiple sclerosis and, finally, disability and cognitive impairment degree was assessed by the expanded disability status scale (EDSS). ${ }^{9}$ According to the multiple sclerosis type, the patients were classified in three subgroups: i) relapsing remitting form, i.e. unpredictable attacks followed by slow remission (symptoms and signs improvement); ii) secondary progressive form, i.e. progressive form with initially relapsing remitting course; and iii) primary progressive form, with gradually worsening of symptoms. ${ }^{8,9}$ The study was approved by the ethical committee of our institution, and carried out in accordance with the principles of the Helsinki Declaration. All patients gave their written consent for ultrasonographic examination.

\section{Ultrasound evaluation of cere- brospinal venous outflow}

All subjects underwent an ECD examination of the cerebrospinal venous flow. In order to reduce bias, all the ECD evaluations were performed by a single, expert ultrasonographer for each center. The examination was performed with the patients in the sitting and supine positions. The physician tried not to exert any pressure on the internal jugular veins because of their collapsibility. We investigated the morphology and hemodynamics of the internal jugular veins by means of high resolution Bmode ultrasounds (MyLab Vinco echo-colorDoppler System, Esaote spa, Genova, Italy, equipped with 2.5 and $7.5-10 \mathrm{MHz}$ probes). We tried to detect venous anatomical abnormalities such as septa/valve malformations and membranes which are able to influence the hemodynamics of cerebral veins in these patients. According to Ciccone et al. ${ }^{10,11}$ we defined: septa/valve malformations as valvular abnormalities of the veins able to create an obstacle to the blood flow in the internal jugular veins and/or brachiocephalic/anonymous trunk junction; membranes as membranes are able to occlude cerebral veins. Hemodynamics parameters and symbols considered in our

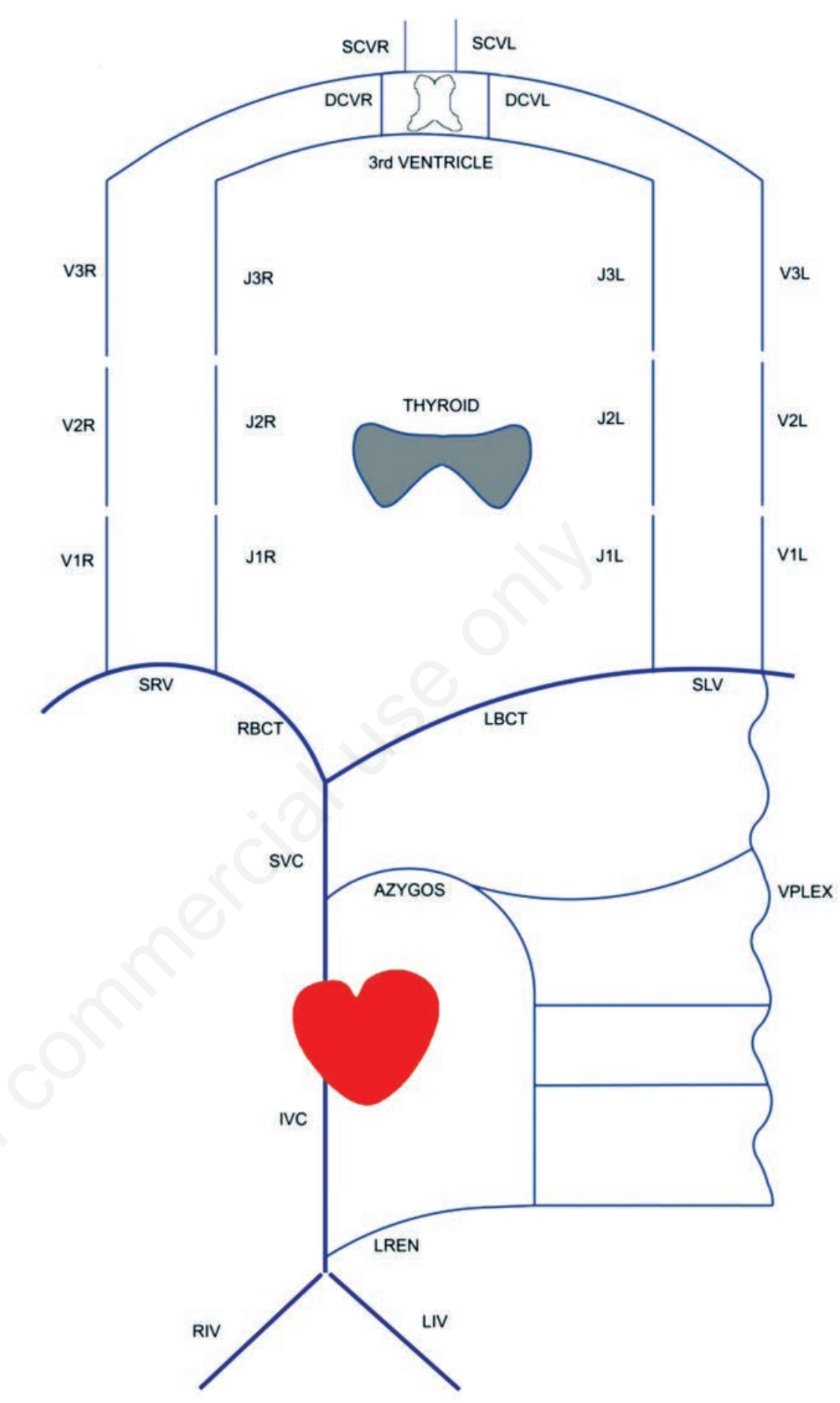

Figure 1. Map scheme for an echo-color-Doppler report. RDCV, right deep cerebral veins; LDCV, left deep cerebral veins; RVV, right vertebral vein; LVV, left vertebral vein; Vplex, vertebral plexus; RBCT, right common-brachiocephalic trunk anonymous; LBCT, left common-brachiocephalic trunk anonymous; RSVC, right superior cava vein; LSVC, left superior cava vein; IVC, inferior cava vein; AZYGOS, Azygos vein; LREN, left renal vein; RIV, right iliac vein; LIV, left iliac vein; J1R, lower right internal jugular vein; J2R, middle right internal jugular vein; J3R, upper right internal jugular vein; J1L, lower left internal jugular vein; J2L, middle left internal jugular vein; J3L, upper left internal jugular vein; V1R, lower right vertebral vein; V2R, middle right vertebral vein; V3R, upper right vertebral vein; V1L, lower left vertebral vein; V2L, middle left vertebral vein; V3L, upper left vertebral vein; SRV, subclavian right vein; SLV, subclavian left vein; SCV, superior cava vein. 
work were those used in the glossary of the ISNVD consensus and position statement. ${ }^{3}$

Patients made a neck rotation test which is still not included in the position statement. ${ }^{3}$ This maneuver was described in the 80's and showed that rotation in normal people opens the IJV from one side and close from the other. ${ }^{12}$ The test consists in the rotation of the head to the outside and inwards, at the same time we check the changes of ultrasound images of the internal jugular veins and vertebral veins to highlight the venous compressive syndrome of these vessels. We defined: physiological internal jugular vein flow when the cross area is more than $6.0 \mathrm{~mm}^{2}$ without flow block or reflux; white compression when the internal jugular vein flow blocked by fully compression; black compression when the internal jugular vein cross area less than $6.0 \mathrm{~mm}^{2}$ by severe compression. ${ }^{13}$

Neurological MS clinical assessment Kurtzke's EDSS ${ }^{9}$ was adopted to evaluate neurological impairments of multiple sclerosis patients. It evaluated the functionality of eight neurologic functional systems [pyramidal (ability to walk); cerebellar (coordination); brain stem (speech and swallowing); sensory (touch and pain); bowel and bladder functions; visual; mental; other (includes any other neu- rological findings due to multiple sclerosis) each scored from 0 (preserved function) to 6 (totally impaired function)]. Furthermore, a judgment about the autonomy of the patients fulfilled the EDSS final score. The final EDSS ranged from 0 (no symptoms) to 10 (death due to multiple sclerosis).

\section{The venous map}

The EDC map scheme is a stylized diagram of the veins draining the brain and spinal cord (Figure 1). The internal jugular and the vertebral veins, on the map, are divided into three segments. The proximal segments are J1-V1, the medium segments are J2-V2 and the distal are J3-V3. J1, J2 and J3 are the segments of internal Jugular veins. J1 segments goes from the confluence into subclavian vein to the inferior level of thyroid. J2 segment goes from inferior level of thyroid to the jugular point; this point is when the vein crosses the bifurcation of carotid arteries. J3 segment goes from the jugular point to the higher point detectable by ECD. V1,V2 and V3 are the segments of vertebral veins. V1 segment goes from the confluence into subclavian vein to the point in which the vertebral vein goes up from vertebral channel. V2 segment goes from this last point to C3 bone. V3 segment above this level. The veins are represented by blue lines and on them are reported the morphological and the hemodynamic symbols that correspond to the assessment of ECD. With color red are reported the symbols of the ECD assessment in supine position; with green color the symbols in the upright position. When the sonographer finds a morphological anomaly or a hemodynamic pathological flow he immediately puts the equivalent symbol on the correspondent point of the map (Figure 1). A legend with all the symbols are close to the map and you can pick which you need to put on the map by using the mouse.

\section{The Mem-net program}

To enter in the Mem-net program, of NEO on CCSVI platform, you must register yourself putting yours personal ID and password to have the access to the program. You can insert a new patient in the archive and automatically the program creates its ID number for the privacy. Ones entering in the program you can put all the personal and clinical data of the patient. Then you can enter in the hemodynamic page where you find the symbols and the Mem-net map for ECD report (Figure 2). After saved the

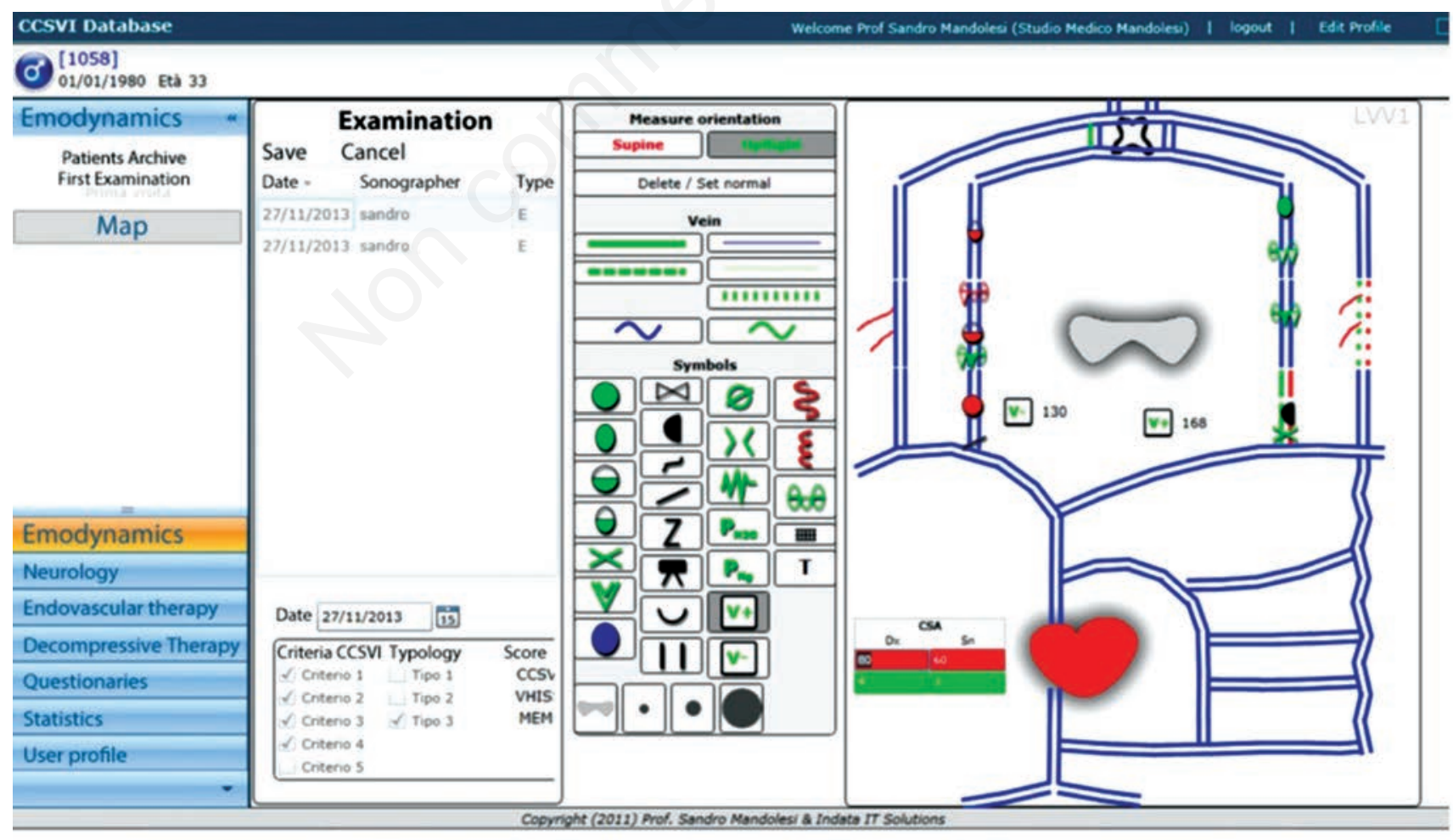

Figure 2. Digitalize the map (Mem-net). CCSVI, chronic cerebrospinal venous insufficiency; CSA, cross-sectional area; VHISS, venous hemodynamic insufficiency severity score; MEM, hemodynamic morphological map. 
map of ECD assessment you can print the map (Figure 3) and the written report (Figure 4).

The MEM-net report shortens the time of ECD written reporting. The Mem-net program allows collecting all the ECD examination data and, by its algorithm, making uniform the report. The input of the hemodynamic and morphological symbols on Memnet map automatically allows the follows prints: ECD morphological hemodynamic map with the legend of the symbols, the written reporting, the criteria we founded, the CCSVI score, the venous hemodynamic insufficiency severity score (VHISS) score, the CCSVI types (type 1, type 2 or type 3 ), the MS clinical types [relapsing-remitting (RR), secondary progressive (SP), primary progressive (PP)], the EDSS, the years of disease duration, the first symptom, age and sex, the identification code for the privacy of the patient. You can also use the statistic area for general or clinical data analysis of patients. This software makes faster, easier and topographic ECD report of CCSVI, reduces human error, standardize the ECD report, by the shared symbols of consensus conference, write the report of ECD assessment, prints automatically the report, saves all hemodynamic and morphological findings in its data base, clinical and hemodynamic follow-up become faster and easier.

\section{Statistical analysis}

Mem-net ECD archives are a web complex database based on structured query language (SQL) software architecture. All stored medical information are classified in SQL table. After registration, authorized users may perform frequency statistics by using preconfigured query in the web page dedicated to statistics. Every Mem-net collaborator may control her/his data choosing many parameters of their patients (sex, age, pathologies, symptoms, illness duration, etc). The central server elaborates the requests building a specific query. Mem-net software elaborate data giving a detailed printable report. In real time, this function permits to all Mem-net collaborators to know statistical situation of her/his patients with related patients from other center.

Mem-net NEO has an epidemiological and statistical team that perform periodical analysis on data quality and medical research in collaborations with Mem-net centers. To perform this activity, data are periodically extracted from Mem-net SQL server database into Microsoft database format (MDB) or in Microsoft excel office format (XLS). These MDB or XLS files are completely compatible with all statistical software programs (BMDP, SPSS, EPI-INFO, etc.). Analysis of variance (ANOVA) or analysis of co-variance (ANCO-
VA) are performed on continuous variables to determine statistical significance between or within data groups. On the same time, logistic analysis is performed on categorical variables by non parametric statistical tests.

These activities permit the Mem-net database maintenance for showing: i) each patient correct and complete information list; ii) epidemiological changes inside etiologic and pathological evolution of illness; iii) unknown and new typical patient profile with specific characteristics.

\section{Results}

We analyzed data of only four centers on seven (Rome, Bari, Cagliari and Benevento). Total patients number was 1109 , mean age $46.0 \pm 13.4$ [male 422 (38.05\%); female 687 (61.95\%)]. CCSVI positive patients were 937 (84.49\%), CCSVI negative patients were 172 (15.51\%). The CCSVI type 1 subjects were 530 (56.56\%), CCSVI type 2 subjects were 20 (2.13\%), CCSVI type 3 subjects were 387 (41.30\%). We found $800(85.38 \%)$ patients

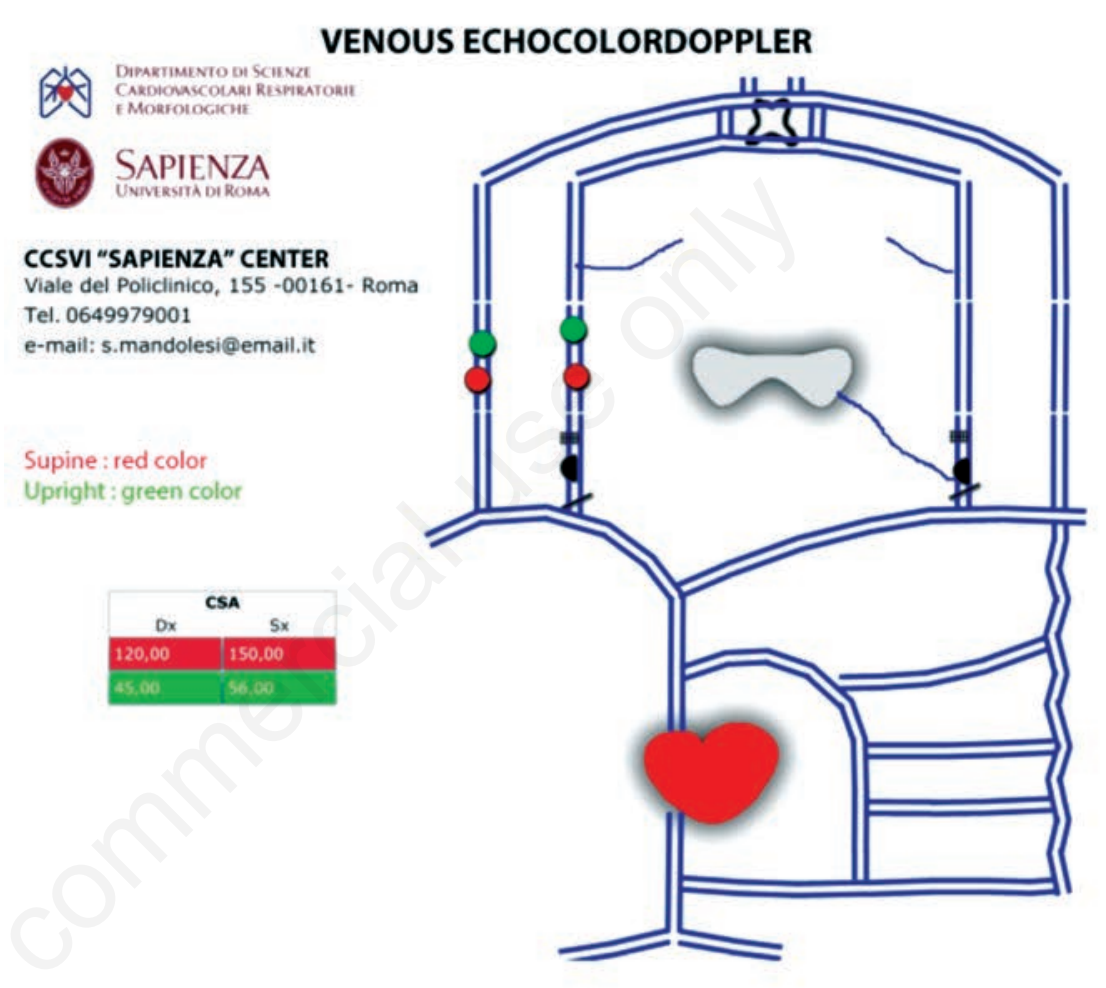

\begin{tabular}{|c|c|}
\hline $\begin{array}{l}\text { Zamboni Criteria } \\
\square \text { Criterion 1 } \\
\square \text { Criterion 2 } \\
\text { Criterion 3 } \\
\square \text { Criterion 4 } \\
\text { Criterion 5 }\end{array}$ & $\begin{aligned} & \text { CCSVI TYPE } \\
& \checkmark \text { Type } 1\end{aligned}$ \\
\hline $\begin{array}{l}\text { ID Patient: } 756 \quad \text { Sex: } F \\
\text { Age: } 33 \\
\text { Clinical Type: SP } \\
\text { First symptom: Optical Neuryte left eye } \\
\text { Duration: } 13 \text { years }\end{array}$ & $\begin{array}{l}\text { Score } \\
\text { CCSVI: } 1 \quad \text { CCSVI is positive if } \geq 2 \text { Criteria } \\
\text { VHISS: } 2 \\
\text { MEM: } \quad 15 \\
\text { Date: } 01 / 27 / 2013 \\
\text { Sonographer: Prof Sandro Mandolesi }\end{array}$ \\
\hline
\end{tabular}

Figure 3. Report printing of the echo-color-Doppler examination with hemodynamic and morphological symbols. CCSVI, chronic cerebrospinal venous insufficiency; CSA, cross-sectional area; SP, secondary progressive; EDSS, expanded disability status scale; VHISS, venous hemodynamic insufficiency severity score; MEM, hemodynamic morphological map; Dx, left; Sx, right. 
Sapienza CCSVI Center

Viale del Policlinico, 155-00161-Roma

Tel. 0649979021

e-mail: s.mandolesi@email.it

Dear $\mathrm{Mr} / \mathrm{Ms}$

ID [345]

The EchoColorDoppler assessment of veins draining your brain shows enough Zamboni's criteria
for Chronic Cerebro-Spinal Venous Insufficiency diagnosis.

In attached ECD map you can find the anatomical anomalies and hemodynamic pathological conditions.

We suggest venography examination and if necessary angioplasty of these veins.

12.29 .2010 with criterion 1; 725 (77.37\%) with criterion 2; 519 (55.39\%) with criterion 3; 483 (51.55\%) with criterion $4 ; 88(9.39 \%)$ with criterion 5 . The VHISS mean score was 3.8 ; the CCSVI mean score was 2.8; the MEM mean score was 34.7; the EDSS mean score was 4.5; the disease mean duration was $12.5 \pm 5.7$ years. Multiple sclerosis clinical types were divided as follows: RR patients were 449 (47.92\%), SP patients were 144 (15.37\%), PP patients were 72 (7.68\%) (Table 1).

\section{Discussion}

In 2011 Laupacis and colleagues in Canada identified a strong and statistically significant relationship [odds ratio (OR) 13.5 ; $95 \%$ confidence interval (CI) 2.6-71.4] between CCSVI and MS, but there was extensive, unexplained heterogeneity across studies $(I 2=89 \%) .{ }^{14}$ In 2013 Tsivgoulis et al. published a meta-analysis of case-control studies, he identified 19 eligible studies including 1250 patients with MS and 899 healthy controls. The pooled analysis showed that CCSVI was associated with MS (OR 8.35; 95\% CI 3.44-20.31; $\mathrm{P}<0.001$ ) with considerable heterogeneity across studies $(I 2=80.1 \%)$. The greatest factor contributing to this heterogeneity appears to be the involvement of investigators in other publications supporting endovascular procedures as a novel MS treatment. ${ }^{15}$

In 2013 Comi et al. published an observational case-control study of the prevalence of chronic cerebrospinal venous insufficiency in multiple sclerosis: results from the CoSMo study. ${ }^{16}$ The study involved 35 MS centers across Italy and included 1874 subjects aged 18-55. 1767 (94\%) were evaluable: $1165 \mathrm{MS}$ patients, 226 patients with other neurological diseases (ONDs) and 376 healthy controls (HCs). CCSVI prevalence was 3.26\%, 3.10\% and $2.13 \%$ for the MS, OND and HC groups, respectively. The conclusions were that CCSVI

Figure 4. Written report of the echo-color-Doppler examination.

Table 1. Chronic Cerebrospinal Venous Insufficiency National Epidemiological Observatory statistical analysis from four centers.

\begin{tabular}{|c|c|c|c|c|c|}
\hline Patients' characteristics & No. & CCSVI classification & No. (\%) & Scores & No. (\%) \\
\hline Total number of patients & 1109 & CCSVI type 1 & $530(56.56 \%)$ & Mean VHISS & 3.8 \\
\hline Total Mem-net patients & 1109 & CCSVI type 2 & $20(2.13 \%)$ & Mean CCSVI score & 2.8 \\
\hline Mean age (years) & $46.0 \pm 13.4$ & CCSVI type 3 & $387(41.30 \%)$ & Mean MEM score & 34.7 \\
\hline Male & 422 & Criterion 1 & $800(85.38 \%)$ & Mean EDSS score & 4.5 \\
\hline Female & 687 & Criterion 2 & $725(77.37 \%)$ & Mean disease duration (years) & $12.5 \pm 5.7$ \\
\hline Positive CCSVI & 937 & Criterion 3 & $519(55.39 \%)$ & $\mathrm{RR}$ & $449(47.92 \%)$ \\
\hline \multirow[t]{3}{*}{ Negative CCSVI } & 172 & Criterion 4 & $483(51.55 \%)$ & SP & $144(15.37 \%)$ \\
\hline & & Criterion 5 & $88(9.39 \%)$ & PP & $72(7.68)$ \\
\hline & & & & CIS & $2(0.21 \%)$ \\
\hline
\end{tabular}

CCSVI, chronic cerebrospinal venous insufficiency; VHISS, venous hemodynamic insufficiency severity score; MEM, hemodynamic morphological map; EDSS, expanded disability disease score; RR, relapsing-remitting; SP, secondary progressive; PP, primary progressive; VHISS, venous hemodynamic insufficiency severity score. 
is not associated with MS. All centers were with conflicting interests with pharmaceutical company, used different ECD devices and their sonographers were not approved by Zamboni University Vascular Department.

To overcome that incredible difference in results between neuro-sonographers and vascular sonographers we created a tool and a method of CCSVI data collection (CCSVI national epidemiological Observatory). The project involves the construction of a CCSVI diagnostic centers network throughout the country, linked all together on internet. The venous ECD for the diagnosis of CCSVI is complex, for that we realized a reporting system on a digitalized map (Mem-net) to easily store the data of this ultrasound assessment. To join the Observatory network the sonographers must confirm either the attendance of a training course at the Vascular Diseases Center of Prof. Zamboni or in a center affiliated than an Observatory course for the use of the venous hemodynamics morphological map (Mem-net) that permit us to value their adequate ECD assessment reproducibility. ${ }^{17,18}$

\section{Conclusions}

The ECD data are collected by the same standard procedure (map report) that permit an ECD assessment reproducibility and eliminate the possibility of heterogeneity. The MEM-net statistical data analysis program allows having homogeneous samples in short time. The CCSVI-NEO database and Mem-net software may be useful medical and researching tools for recording, storing, analyzing and studying ECD and vascular data all over the world. We hope that Italian experience will be the first step for reaching European and international CCSVI-NEO database. We hope that some critical issue in the Mem-net can be improved in the future and that it becomes a tool widely distributed for standardized analysis of the chosen 90 statistical items for CCSVI.

Finally, preliminary data of NEO show an elevated prevalence of CCSVI in MS.

\section{References}

1. Zivadinov R, Bastianello S, Dake MD, et al. Recommendations for multimodal noninvasive and invasive screening for detection of extracranial venous abnormalities indicative of chronic cerebrospinal venous insufficiency: a position statement of the International Society for Neurovascular Disease. J Vasc Interv Radiol 2014 [Epub ahead of print].

2. Zamboni P, Galeotti R, Menegatti E, et al. Chronic cerebrospinal venous insufficiency in patients with multiple sclerosis. $\mathrm{J}$ Neurol Neurosurg Psychiatry 2009;80:3929.

3. Zamboni P, Menegatti E, Galeotti R, et al. The value of cerebral Doppler venous haemodynamics in the assessment of multiple sclerosis. J Neurol Sci 2009;282:21-7.

4. Zamboni P. Chronic cerebrospinal venous insufficiency. Int Angiol 2010;29:91-2.

5. Zamboni P, Menegatti E, WeinstockGuttman B, et al. The severity of chronic cerebrospinal venous insufficiency in patients with multiple sclerosis is related to altered cerebrospinal fluid dynamics. Funct Neurol 2009;24:133-8.

6. Zamboni P, Menegatti E. Doppler haemodynamics of vertebral venous return. Curr Neurovasc Res 2008;5:260-5.

7. Polman CH, Reingold SC, Edan G, et al. Diagnostic criteria for multiple sclerosis: 2005 revisions to the McDonald criteria. Ann Neurol 2005;58:840-6.

8. McDonald WI, Compston A, Edan G, et al. Recommended diagnostic criteria for multiple sclerosis: guidelines from the International Panel on the diagnosis of multiple sclerosis. Ann Neurol 2001;50:121-7.

9. Kurtzke JF. Rating neurological impairment in multiple sclerosis: an expanded disability scale (EDSS). Neurology 1983;33:1444-52.

10. Ciciarello F, Mandolesi S, Galeandro AI, et al. Age-related vascular differences among patients suffering from multiple sclerosis. Curr Neurovasc Res 2014;11:23-30.
11. Ciccone MM, Galeandro AI, Scicchitano P, et al.. Multigate quality Doppler profiles and morphological/hemodynamic alterations in multiple sclerosis patients. Curr Neurovasc Res 2012;9:120-7.

12. Muhammad JK, Pugh ND, Boden L, et al. The effect of head rotation on the diameter of the internal jugular vein: implications for free tissue transfer. J Craniomaxillofac Surg 2001;29:214-8.

13. Mandolesi S, Manconi E, Niglio T, et al. Incidence of anatomical compressions of the internal jugular veins with full block of their flow in patients with chronic cerebro-spinal venous insufficiency and multiple sclerosis. In: Allegra C, Antignani PL, eds. Proc. $21^{\text {st }}$ EUROCHAP-IUA - European Chapter Congress of the International Union of Angiology. Sept 28-0ct 1, 2013, Rome, Italy. Turin: Ed. Minerva Medica; 2013. pp 12-17.

15. Laupacis A, Lillie E, Dueck A, et al. Association between chronic cerebrospinal venous insufficiency and multiple sclerosis: a meta-analysis. CMAJ 2011;183:E1203-12.

16. Tsivgoulis G. Chronic cerebrospinal venous insufficiency and multiple sclerosis: a comprehensive meta-analysis of case-control studies. Ther Adv Neurol Disord 2013;25:269-79.

17. Comi G, Battaglia MA, Bertolotto A, et al. Observational case-control study of the prevalence of chronic cerebrospinal venous insufficiency in multiple sclerosis: results from the CoSMo study. Mult Scler 2013;19:1508-17.

18. Dolic K, Marr K, Valnarov V, et al. Sensitivity and specificity for screening of chronic cerebrospinal venous insufficiency using a multimodal non-invasive imaging approach in patients with multiple sclerosis. Funct Neurol 2011;26:205-14.

19. Menegatti E, Genova V, Tessari M, et al. The reproducibility of colour Doppler in chronic cerebrospinal venous insufficiency associated with multiple sclerosis. Int Angiol 2010;29:121-6. 